\title{
Erratum to: Three decades of publishing research in population economics
}

\author{
Alessio J. G. Brown ${ }^{1}$ - Klaus F. Zimmermann ${ }^{1,2}$
}

Published online: 19 October 2016

C) Springer-Verlag Berlin Heidelberg 2016

\section{Erratum to: J Popul Econ}

\section{DOI 10.1007/s00148-016-0620-1}

The original version of this paper unfortunately contained an error in table 3 . The correct Table 3 is given below:

The online version of the original article can be found at http://dx.doi.org/10.1007/s00148-016-0620-1.

$\triangle$ Klaus F. Zimmermann

klaus.f.zimmermann@gmail.com

1 UNU-MERIT, Maastricht, The Netherlands

2 Harvard University, Cambridge, MA, USA 
Table 3 Kuznets Prize winners and winning papers

\begin{tabular}{|c|c|c|c|}
\hline Years & Author(s) & Title & Volume, Issue \\
\hline 2017 & $\begin{array}{l}\text { Semih Tumen and } \\
\text { Binnur Balkan }\end{array}$ & $\begin{array}{l}\text { Immigration and prices: quasi-experimental } \\
\text { evidence from Syrian refugees in Turkey }\end{array}$ & 2016, 29(3): 657-686 \\
\hline 2016 & $\begin{array}{l}\text { Loren Brandt, Aloysius } \\
\text { Siow and Hui Wang }\end{array}$ & $\begin{array}{l}\text { Compensating for unequal parental } \\
\text { investments in schooling }\end{array}$ & $2015,28(2): 423-462$ \\
\hline 2015 & Haoming Liu & $\begin{array}{l}\text { The quality-quantity trade-off: evidence } \\
\text { from the relaxation of China's } \\
\text { one-child policy }\end{array}$ & 2014, 27 (2): 565-602 \\
\hline 2014 & Paolo Masella & National identity and ethnic Diversity & 2013,26 (2): 437-454 \\
\hline 2010-2012 & $\begin{array}{l}\text { Richard W. Evans, } \\
\text { Ying-yao Hu and } \\
\text { Zhong Zhao }\end{array}$ & $\begin{array}{l}\text { The fertility effect of catastrophe: } \\
\text { US hurricane births }\end{array}$ & 2010, 23 (1): 1-36 \\
\hline 2007-2009 & $\begin{array}{l}\text { Makoto Hirazawa and } \\
\text { Akira Yakita }\end{array}$ & $\begin{array}{l}\text { Fertility, child care outside the home, } \\
\text { and pay-as-you-go social security }\end{array}$ & 2009, 22 (2): 565-583 \\
\hline 2004-2006 & Jinyoung Kim & $\begin{array}{l}\text { Sex selection and fertility in a dynamic } \\
\text { model of conception and abortion }\end{array}$ & 2005,18 (1): 41-67 \\
\hline $2001-2003$ & $\begin{array}{l}\text { Olympia Bover and } \\
\text { Manuel Arellano }\end{array}$ & $\begin{array}{l}\text { Learning about migration decisions from } \\
\text { migrants: Using complementary datasets } \\
\text { to model intra-regional migrations in Spain }\end{array}$ & 2002,15 (2): $357-380$ \\
\hline 1998-2000 & David C. Ribar & $\begin{array}{l}\text { The socioeconomic consequences of } \\
\text { young women's childbearing: } \\
\text { Reconciling disparate evidence }\end{array}$ & 1999,12 (4): $547-565$ \\
\hline 1995-1997 & James R. Walker & $\begin{array}{l}\text { The effect of public policies on recent } \\
\text { Swedish fertility behavior }\end{array}$ & 1995,8 (3): $223-251$ \\
\hline
\end{tabular}

